Mathematical Research Letters 7, 643-650 (2000)

\title{
EXTENSION AND REPRESENTATION OF DIVERGENCE-FREE VECTOR FIELDS ON BOUNDED DOMAINS
}

Tosio Kato, Marius Mitrea, Gustavo Ponce, and Michael Taylor

\section{Introduction}

Let $\Omega \subset \mathbb{R}^{n}$ be a bounded, connected domain, with $b+1$ boundary components, $\partial \Omega=\Gamma_{0} \cup \cdots \cup \Gamma_{b}$. Say $\mathcal{O}_{0}, \ldots, \mathcal{O}_{b}$ are the connected components of $\mathbb{R}^{n} \backslash \bar{\Omega}, \mathcal{O}_{0}$ being the unbounded component, and $\Gamma_{j}=\partial \mathcal{O}_{j}$. If $b>0$, pick $y_{j} \in \mathcal{O}_{j}, 1 \leq j \leq b$, and set

$$
g_{j}(x):=\frac{x-y_{j}}{\left|x-y_{j}\right|^{n}}, \quad x \in \mathbb{R}^{n} \backslash\left\{y_{j}\right\} .
$$

Let $H^{s, p}$ denote the usual scale of $L^{p}$-Sobolev spaces, and denote by div and Div the divergence of 1-tensors (i.e., vector fields) and 2-tensors, respectively.

Given a divergence-free vector field $u$ on $\Omega$, possessing a certain regularity, e.g., $u \in H^{s, p}(\Omega)$, we want to investigate two closely related problems. One is to extend $u$ to a divergence-free vector field $\tilde{u}$ defined on a neighborhood of $\bar{\Omega}$ (in fact, defined on $\mathbb{R}^{n} \backslash\left\{y_{j}\right\}$ ), such that $\tilde{u}$ is as smooth as $u$. The second is to produce an anti-symmetric 2-tensor field $v$ such that

$$
u=\operatorname{Div} v+\sum_{j \geq 1} \lambda_{j} g_{j}
$$

for some constants $\lambda_{j} \in \mathbb{R}$, and such that

$$
v \in H^{s+1, p}(\Omega) .
$$

Note that if we can solve the second problem, then we need merely extend $v$ to $\tilde{v}$ (a familiar task) and set

$$
\tilde{u}=\operatorname{Div} \tilde{v}+\sum \lambda_{j} g_{j}
$$

\footnotetext{
Received February 10, 2000.

2000 Mathematics Subject Classification. Primary: 53A45, 58A14; Secondary: 35J25,

Key words and phrases. Structure of divergence-free fields, extension operators, Lipschitz

Work supported in part by NSF.
} $42 \mathrm{~B} 20$ domains. 
Note that we can arrange that $\tilde{v}$ be supported on an arbitrarily small neighborhood of $\bar{\Omega}$.

We expect these results to be useful in a variety of situations such as in the study of the motion of incompressible fluids and in elasticity. For example, consider the boundary value problem (BVP) for an incompressible fluid (ideal or viscous) in $\Omega$. One could attempt to estimate the influence of the boundary on the solution by removing a portion of the boundary, extending the data to the new domain, and comparing the solution of this new BVP (or initial value problem if the whole boundary is removed) to the original one.

Another application of our results is the solution of a useful subspace (complex) interpolation problem. Specifically, if $\Omega$ is a bounded Lipschitz domain and $L_{\sigma}^{p}(\Omega)$ stands for the (closed) subspace of $L^{p}$-vector fields which are also divergence free in $\Omega$, then

$$
\left[L_{\sigma}^{p_{0}}(\Omega), L_{\sigma}^{p_{1}}(\Omega)\right]_{\theta}=L_{\sigma}^{p^{*}}(\Omega)
$$

provided $1<p_{0}, p_{1}<\infty, 0<\theta<1$, and $1 / p^{*}=(1-\theta) / p_{0}+\theta / p_{1}$. Indeed, ultimately this is a consequence of the fact that the operator

$$
T: H^{1, p}(\Omega) \times \mathbb{R}^{b} \rightarrow L_{\sigma}^{p}(\Omega), \quad\left(v,\left\{\lambda_{j}\right\}_{j}\right):=\operatorname{Div} v+\sum_{j} \lambda_{j} g_{j}
$$

is onto. Other variants (e.g., for more regular fields and domains) are, of course, possible.

The plan of the paper is as follows. We will first treat the case where $\partial \Omega$ is smooth, in $\S \S 2-3$, and then extend these results to cases where $\partial \Omega$ has minimal regularity. In $\S 2$ we treat divergence-free $u \in L^{p}(\Omega), 1<p<\infty$. In this case we first solve the extension problem, obtaining $\tilde{u} \in L_{\text {loc }}^{p}\left(\mathbb{R}^{n} \backslash\left\{y_{j}\right\}\right)$, and use this to find $v \in H^{1, p}(\Omega)$ satisfying (1.2).

In $\S 3$ we treat divergence-free $u \in H^{s, p}(\Omega)$, for $s \geq 0$ and $1<p<\infty$. Here we obtain the representation (1.2) as a consequence of the Hodge decomposition for differential forms on bounded domains, as worked out by Friedrichs and by Morrey and Eells (cf. [Mor], Chapter 7), together with other known results on elliptic boundary problems satisfying the Lopatinski condition. While the results of $\S 3$ formally contain those of $\S 2$, it is convenient to have $\S 2$ in order to dispose quickly of a technical point that arises when $H^{s, p}(\Omega)$ is not contained in $H^{1,2}(\Omega)$.

In $\S 4$ we extend the results of $\S 3$ to the situation where $\partial \Omega$ is of class $C^{k, \sigma}$, for some $k \in \mathbb{Z}^{+}$and $\sigma \in(0,1]$ (as long as $k+\sigma \geq 1$ ). We accomplish this via a change of variables and use of the Hodge star operator. Section 5 discusses further extensions, such as to fields of class $C^{k-1, \sigma}$ and to exterior domains. 


\section{2. $L^{p}$-fields}

Let $\Omega \subset \mathbb{R}^{n}$, be as in $\S 1$, and assume $\partial \Omega$ is smooth. As stated in the introduction, we start with an extension result.

Proposition 2.1. Let $B \subset \mathbb{R}^{n}$ be a smoothly bounded open set containing $\bar{\Omega}$. Given $1<p<\infty$ and a vector field $u \in L^{p}(\Omega)$ such that $\operatorname{div} u=0$, there exist $\lambda_{j} \in \mathbb{R}$ (uniquely determined by $u$ ) and there exists $u^{\#} \in L^{p}\left(\mathbb{R}^{n}\right)$ such that supp $u^{\#} \subset \bar{B}$, div $u^{\#}=0$ on $\mathbb{R}^{n}$, and

$$
u^{\#}=u-\sum \lambda_{j} g_{j} \quad \text { on } \Omega .
$$

Furthermore, we can take $u^{\#}=T u$ with $T: L^{p}(\Omega) \rightarrow L^{p}\left(\mathbb{R}^{n}\right)$ bounded.

Proof. Denote by $\nu$ the outward unit normal to $\partial \Omega$, by $d \sigma$ the surface measure on $\partial \Omega$ and fix $\lambda_{j} \in \mathbb{R}$ such that

$$
f:=\nu \cdot u-\nu \cdot\left(\sum_{j} \lambda_{j} g_{j}\right) \in B_{-1 / p}^{p, p}(\partial \Omega)
$$

satisfies

$$
\int_{\Gamma_{j}} f d \sigma=0, \quad \forall j \geq 1
$$

Here the normal component $\nu \cdot u$, of an $L^{p}$-field $u$ whose divergence is also in $L^{p}$, is defined in the sense of distributions and $B_{s}^{p, q}$ stands for the Besov scale. Note that (2.3) determines the numbers $\lambda_{j}$ uniquely and that $\sum\left|\lambda_{j}\right| \leq C\|u\|_{L^{p}(\Omega)}$. Moreover, as a result of the divergence theorem, $\int_{\Gamma_{0}} f d \sigma=0$ also.

Set $D:=B \backslash \bar{\Omega}$. Also, extend $f$ to $\partial D$ by zero on $\partial B$. Then we can solve the Neumann problem

$$
\left\{\begin{array}{l}
\Delta w=0 \text { in } D \\
\frac{\partial w}{\partial \nu}=f \in B_{-1 / p}^{p, p}(\partial D), \\
w \in H^{1, p}(D) .
\end{array}\right.
$$

Since the domain is smooth, this follows from Theorem 4.3.3 on pp. 233-234 of [Tr]. For an extension to the class of Lipschitz domains, the interested reader may also consult $[\mathrm{MT}]$. The function $w$ in (2.4) is uniquely determined if we impose the conditions $\int_{D_{j}} w=0$ where $D_{j}$ are the connected components of $D$.

If we now consider

$$
u^{\#}:=\left\{\begin{array}{l}
u-\sum_{j} \lambda_{j} g_{j} \text { in } \Omega, \\
\nabla w \text { in } D, \\
0 \text { in } \mathbb{R}^{n} \backslash \bar{B},
\end{array}\right.
$$

then $u^{\#} \in L^{p}\left(\mathbb{R}^{n}\right)$, has compact support and, in the sense of distributions, $\operatorname{div} u^{\#}=0$ in $\mathbb{R}^{n}$.

Now we use Proposition 2.1 to establish the following representation result. 
Proposition 2.2. Assume $1<p<\infty$. For any vector field $u \in L^{p}(\Omega)$ satisfying div $u=0$ there exist an antisymmetric 2-tensor field $v \in H^{1, p}\left(\mathbb{R}^{n}\right)$ which satisfies $d v=0$ and numbers $\lambda_{j} \in \mathbb{R}$ such that

$$
u=\operatorname{Div} v+\sum_{j \geq 1} \lambda_{j} g_{j} \text { in } \Omega .
$$

Furthermore, the numbers $\lambda_{j}$ are unique and

$$
\sum_{j \geq 1}\left|\lambda_{j}\right| \leq C\|u\|_{L^{p}(\Omega)}
$$

where $C>0$ depends only on $p, \Omega$ and the collection $\left\{y_{j}\right\}_{j}$. Also, $v$ can be chosen to depend linearly on $u$ and such that $\|v\|_{H^{1, p}\left(\mathbb{R}^{n}\right)} \leq C\|u\|_{L^{p}(\Omega)}$.

Proof. Take $u^{\#}$ as given above and let $\Pi:=\Delta^{-1}$ stand for the harmonic Newtonian potential in $\mathbb{R}^{n}$. Then

$$
\begin{aligned}
u^{\#} & =\Delta \Pi u^{\#}=-d \operatorname{div} \Pi u^{\#}-\operatorname{Div} d \Pi u^{\#} \\
& =-d \Pi \operatorname{div} u^{\#}-\operatorname{Div} d \Pi u^{\#}=-\operatorname{Div} d \Pi u^{\#} .
\end{aligned}
$$

Set now $v:=-d \Pi u^{\#}$ so that $v \in H^{1, p}\left(\mathbb{R}^{n}\right)$ and $\|v\|_{H^{1, p}\left(\mathbb{R}^{n}\right)} \leq C\left\|u^{\#}\right\|_{L^{p}\left(\mathbb{R}^{n}\right)} \leq$ $C\|u\|_{L^{p}(\Omega)}$, by standard Calderón-Zygmund theory (cf., e.g., [St]). Moreover, $d v=0$ and $u^{\#}=\operatorname{Div} v$ in $\mathbb{R}^{n}$, so that $v$ does the job advertised in Proposition 2.2 .

With regard to the uniqueness part, note that if $u=0$ can be decomposed as in (2.6), then $\Delta v=0$, so that $v \in C^{\infty}$. Let $*$ stand for the usual Hodge star isomorphism. Then, for any closed, smooth surface $\gamma_{j} \subset \Omega$, surrounding (sufficiently tightly) $\Gamma_{j}, j \geq 1$, Stokes's theorem gives

$$
0=\int_{\gamma_{j}} *(\operatorname{Div} v)=\lambda_{j} \int_{\gamma_{j}} * g_{j}=c_{n} \lambda_{j}
$$

for some (nonzero) dimensional constant $c_{n}$. Thus, $\lambda_{j}=0$ for each $j \geq 1$, as desired.

We mention one simple consequence of Proposition 2.2, which will be of use in $\S 3$.

Corollary 2.3. For $\Omega$ and $p$ as in Proposition 2.1, given $u \in L^{p}(\Omega)$, div $u=0$ on $\Omega$, there exists a neighborhood $\mathcal{O}$ of $\bar{\Omega}$, and $u_{\nu} \in C^{\infty}(\mathcal{O})$ satisfying $\operatorname{div} u_{\nu}=0$ on $\mathcal{O}$ and $u_{\nu} \rightarrow u$ on $\Omega$ in $L^{p}$-norm.

Proof. Apply a Friedrichs mollifier to the extension $u^{\#}+\sum \lambda_{j} g_{j}$ of $u$ constructed above. 


\section{3. $H^{s, p}$-fields}

Let $\Omega \subset \mathbb{R}^{n}$ be as in $\S 2$. We aim to establish the following result.

Proposition 3.1. Take $p \in(1, \infty)$ and $s \geq 0$. Assume $u \in H^{s, p}(\Omega)$ is a vector field satisfying divu $=0$. Then there exist an antisymmetric 2-tensor field $v \in H^{s+1, p}(\Omega)$ and $\lambda_{j} \in \mathbb{R}$ such that

$$
u=\operatorname{Div} v+\sum_{j \geq 1} \lambda_{j} g_{j}
$$

Corollary 3.2. In the context of the previous result, the vector field $v$ has an extension $\tilde{v} \in H^{s+1, p}\left(\mathbb{R}^{n}\right)$, supported on a small neighborhood of $\bar{\Omega}$, and if $\tilde{u}$ is defined by

$$
\tilde{u}=\operatorname{Div} \tilde{v}+\sum \lambda_{j} g_{j}
$$

then this is a divergence-free extension of $u$ on $\mathbb{R}^{n} \backslash\left\{y_{1}, \ldots, y_{b}\right\}$ (on all of $\mathbb{R}^{n}$ if $b=0)$.

Proof of Proposition 3.1. Proposition 3.1 is related to the Hodge decomposition as follows. If we pass from vector fields to differential forms, we are looking at a 1-form $u$ satisfying $\delta u=0$ and we claim that

$$
u=\delta v+\sum \lambda_{j} h_{j}
$$

where $v$ is a 2 -form and $h_{j}$ are harmonic forms. The underlying Hodge decomposition, in the notation used in Chapter $5, \S 9$ of [Ta], is

$$
u=d \delta G^{R} u+\delta d G^{R} u+P_{h}^{R} u,
$$

valid for general $\ell$-forms on $\Omega$. Here $P_{h}^{R}$ is a projection onto a space $\mathcal{H}_{\ell}^{R}$ of harmonic $\ell$-forms, which has dimension $b$ for $\ell=1$. Since $G^{R}$ is the solution operator for an elliptic boundary problem satisfying the Lopatinski condition, we have

$$
G^{R}: H^{s, p}(\Omega) \longrightarrow H^{s+2, p}(\Omega), \quad s \geq 0,1<p<\infty .
$$

Furthermore, we claim that

$$
\delta u=0 \Longrightarrow d \delta G^{R} u=0 \Longrightarrow u=\delta d G^{R} u+P_{h}^{R} u .
$$

For $u \in H^{1,2}(\Omega)$ this is well known. See, e.g., Theorem 7.7.5 in Chapter 7 of [Mor], or Proposition 9.8 (plus (9.48)) in Chapter 5 of [Ta]. We are now claiming 
this implication holds whenever $u \in L^{p}(\Omega), 1<p<\infty$. To see this (at least for 1-forms), use Corollary 2.3 to produce $u_{\nu} \in C^{\infty}(\bar{\Omega})$ such that $\delta u_{\nu}=0$ and $u_{\nu} \rightarrow u$ in $L^{p}(\Omega)$. By the results cited above,

$$
\delta u_{\nu}=0 \Longrightarrow d \delta G^{R} u_{\nu}=0,
$$

but (3.5) implies $G^{R} u_{\nu} \rightarrow G^{R} u$ in $H^{2, p}(\Omega)$, so $d \delta G^{R} u_{\nu} \rightarrow d \delta G^{R} u$ in $L^{p}(\Omega)$, and we have (3.6).

Thus we have (3.3), with $v=d G^{R} u$. To prove (3.1), pick $\lambda_{j}$ such that the flux of $u-\sum \lambda_{j} g_{j}$ is zero across each boundary component $\Gamma_{j}$. Using this in place of $u$ in (3.4), we have no harmonic form component, and hence (3.6) gives $u-\sum \lambda_{j} g_{j}=\operatorname{Div} v$.

To see that we have no harmonic form component when the flux is zero across each component, we make use of the connection between the Hodge decomposition and topology, which we now recall. First, a Hodge decomposition complementary to $(3.4)$ is

$$
u=d \delta G^{A} u+\delta d G^{A} u+P_{h}^{A} u .
$$

In fact, the Hodge star operator applied to an $\ell$-form decomposed via (3.4) takes it to an $(n-\ell)$-form decomposing via (3.7).

The following is a punch line for Hodge theory. If $\mathcal{H}_{\ell}^{A}$ denotes the range of $P_{h}^{A}$ on $\ell$-forms and $\mathcal{H}_{\ell}^{R}$ the range of $P_{h}^{R}$ on $\ell$-forms, we have

$$
\mathcal{H}_{\ell}^{R} \approx \mathcal{H}_{n-\ell}^{A} \approx \mathcal{H}^{n-\ell}(\bar{\Omega}),
$$

the last space denoting the cohomology of $\bar{\Omega}$ with real coefficients. One also has

$$
\mathcal{H}^{n-\ell}(\bar{\Omega}) \approx \mathcal{H}_{n-\ell}(\bar{\Omega})
$$

the homology group. The cycles $\Gamma_{1}, \ldots, \Gamma_{b}$ provide a basis of $\mathcal{H}_{n-1}(\bar{\Omega})$, which explains the argument in the paragraph above (3.7). In detail, applying the Hodge star operator to $u=\delta d G^{R} u+P_{h}^{R} u$ yields for $u^{b}=* u$ the decomposition

$$
u^{b}=d \delta G^{A} u^{b}+P_{h}^{A} u^{b} .
$$

Now $\int_{\Gamma_{j}} u \cdot \nu d \sigma=\int_{\Gamma_{j}} u^{b}$. We see that $\int_{\Gamma_{j}} d \delta G^{A} u^{b}=0$, by Stokes theorem, and for a harmonic form $h^{A}=P_{h}^{A} u^{b}$, we have

$$
h^{A}=0 \Longleftrightarrow \int_{\Gamma_{j}} h^{A}=0, \quad 1 \leq j \leq b .
$$

For use in $\S 4$, we note the following variant of Corollary 3.2.

Corollary 3.3. Assume $\Omega, s$, and $p$ are as in Proposition 3.1. There is a neighborhood $\mathcal{O}$ of $\bar{\Omega}$ with the following property. Given an $(n-1)$-form $w \in$ $H^{s, p}(\Omega)$ such that $d w=0$ on $\Omega$, there is an extension to an $(n-1)$-form $\tilde{w} \in$ $H^{s, p}(\mathcal{O})$ such that $d \tilde{w}=0$ on $\mathcal{O}$.

Proof. Given such $w$, apply Corollary 3.2 to the 1 -form $u=* w$, obtaining $\tilde{u}$. Then take $\tilde{w}=(-1)^{n-1} * \tilde{u}$. 


\section{Lipschitz and $C^{k, \sigma}$ domains}

We now consider a domain whose boundary has limited regularity, of class $C^{k, \sigma}$, with $k=0,1,2, \ldots$ and $\sigma \in(0,1]$. The case $C^{0,1}$ defines the class of Lipschitz domains. Given a bounded domain $\Omega \subset \mathbb{R}^{n}$ of class $C^{k, \sigma}$, provided $k+\sigma \geq 1$ (i.e., either $k>0$ or $\sigma=1$ ) one can produce a homeomorphism $\varphi: \mathcal{O} \rightarrow \mathcal{O}_{1}$ on a neighborhood $\mathcal{O}$ of $\bar{\Omega}$ such that $\varphi$ and its inverse $\varphi^{-1}$ are of class $C^{k, \sigma}$ and $\bar{\Omega}_{1}=\varphi(\bar{\Omega})$ has $C^{\infty}$ boundary.

The pull-back operation $\varphi^{*}$ on differential forms (whose formula involves firstorder derivatives of $\varphi$ ) takes $L^{p}$-forms to $L^{p}$-forms as long as $\varphi$ is of class $C^{0,1}$, and more generally $\varphi^{*}$ takes $H^{s, p}$-forms to $H^{s, p}$-forms as long as either

$$
s \in \mathbb{Z}^{+} \text {and } k+\sigma \geq s+1,
$$

or

$$
k+\sigma>s+1
$$

Using this we can extend the results of $\S 3$ as follows:

Proposition 4.1. The results of Proposition 3.1 and Corollary 3.2 hold when $\partial \Omega$ is of class $C^{k, \sigma}$, with $k \in \mathbb{Z}^{+}, \sigma \in(0,1]$, as long as either (4.1) or (4.2) hold.

Proof. It suffices to produce an extension $\tilde{u} \in H^{s, p}(\mathcal{O})$ satisfying $\delta \tilde{u}=0$ on some neighborhood $\mathcal{O}$ of $\bar{\Omega}$. Indeed, we can then produce $\Omega_{2}$ with smooth boundary such that $\bar{\Omega} \subset \Omega_{2} \subset \bar{\Omega}_{2} \subset \mathcal{O}$ and apply the results of $\S 3$ to $\left.u\right|_{\Omega_{2}}$.

To accomplish this, we proceed as follows. Consider the $(n-1)$-form $w=* u$, satisfying $d w=0$ on $\Omega$. Pick $\varphi$ as above, mapping $\bar{\Omega}$ bijectively onto the smoothly bounded domain $\bar{\Omega}_{1}$, and take $w_{1}=\left(\varphi^{-1}\right)^{*} w$. Thus $w_{1}$ is an $(n-1)$ form in $H^{s, p}\left(\Omega_{1}\right)$, satisfying $d w_{1}=0$ on $\Omega_{1}$. By Corollary $3.3, w_{1}$ has an

extension $\tilde{w}_{1} \in H^{s, p}\left(\mathcal{O}_{1}\right)$, for some neighborhood $\mathcal{O}_{1}$ of $\bar{\Omega}_{1}$, satisfying $d \tilde{w}_{1}=0$ on $\mathcal{O}_{1}$. Now $\tilde{w}=\varphi^{*} \tilde{w}_{1}$ is an $(n-1)$-form in $H^{s, p}(\mathcal{O})$ satisfying $d \tilde{w}=0$ in $\mathcal{O}$, and agreeing with $w$ on $\Omega$. Hence $\tilde{u}=(-1)^{n-1} * \tilde{w}$ is the desired extension of $u$.

Remark. In particular we extend divergence-free vector fields in $L^{p}(\Omega)$ whenever $\Omega$ is a Lipschitz domain and $1<p<\infty$. The approach taken in $\S 2$ works in this situation, given results established in $[\mathrm{MT}]$ on the Neumann problem, but only for $3 / 2-\varepsilon<p<3+\varepsilon$, for some $\varepsilon=\varepsilon(\Omega)>0$, or when $\partial \Omega$ is $C^{1}$ and $1<p<\infty$. This direct approach via (2.4) also holds when $n=2$ and $4 / 3-\varepsilon<p<4+\varepsilon$, via work of $[\mathrm{MD}]$.

\section{Further extensions}

We have analogues of Proposition 4.1 when the divergence-free vector field $u$ belongs to $C^{k-1, \sigma}(\bar{\Omega})$, given $k \geq 1$ and $\sigma \in(0,1)$. When $\partial \Omega$ is of class $C^{k, \sigma}$, one 
has (3.1) for some $v \in C^{k, \sigma}(\bar{\Omega})$ and then $\tilde{u}$ in (3.2) can be chosen to belong to $C_{\text {loc }}^{k-1, \sigma}\left(\mathbb{R}^{n} \backslash\left\{y_{j}\right\}\right)$. To establish this we need merely note that, parallel to (3.5), one has (for smoothly bounded $\Omega$ )

$$
G^{R}: C^{\ell, \sigma}(\bar{\Omega}) \longrightarrow C^{\ell+2, \sigma}(\bar{\Omega}), \quad \ell \in \mathbb{Z}^{+}, 0<\sigma<1,
$$

and that $\varphi^{*}$ and $\left(\varphi^{-1}\right)^{*}$ preserve the category of differential forms of class $C^{k-1, \sigma}$.

Also, we can extend a divergence-free field $u$ on an exterior domain $\Omega=$ $\mathbb{R}^{n} \backslash K$, where $K$ is compact and $\partial K$ of class $C^{k, \sigma}$. Indeed, take a ball $B \supset K$, set $\Omega_{0}=B \backslash K$, apply Proposition 4.1 to $\left.u\right|_{\Omega_{0}}$, and then merely make the extension of $u$ effective on $K$.

Finally, an inspection of the arguments in the previous sections shows that the extension property alluded to above also holds on subdomains of a smooth manifold $M$, equipped with a sufficiently smooth metric tensor.

\section{Acknowledgments}

Thanks are due to the referee for the careful reading and some useful suggestions.

\section{References}

[MD] D. Mitrea, The classical layer potentials for two dimensional Lipschitz domains, preprint (2000).

[MT] M. Mitrea and M. Taylor, Potential theory on Lipschitz domains in Riemannian manifolds: Sobolev-Besov space results and the Poisson problem, J. Funct. Anal. 176 (2000), $1-79$.

[Mor] C. B. Morrey, Multiple Integrals in the Calculus of Variations, Springer-Verlag, New York, 1966.

[St] E. Stein, Singular Integrals and Differentiability Properties of Functions, Princeton University Press, 1970.

[Ta] M. Taylor, Partial Differential Equations, Vols. 1-3, Springer-Verlag, New York, 1996.

[Tr] H. Triebel, Theory of Function Spaces, Birkhauser, Basel, 1983.

Tosio Kato (Deceased), Department of Mathematics, University of California Berkeley, Berkeley, CA 94720, USA.

Department of Mathematics, University of Missouri at Columbia, Columbia, MO 65211, USA.

E-mail address: marius@math.missouri.edu

Department of Mathematics, University of California Santa Barbara, Santa BarBARA, CA 93106, USA.

E-mail address: ponce@math.ucsb.edu

Department of Mathematics, University of North Carolina, Chapel Hill, NC 27599, USA

E-mail address: met@math.unc.edu 